\title{
Der Wald als Kulturphänomen - von der Mythologie zum Wirtschaftsobjekt
}

Unsere heutigen Wälder sind mit wenigen Ausnahmen Kulturphänomene, d.h., sie sind vom menschlichen Denken, Planen und Wollen geprägt. Der Wald, der uns landläufig auf Spaziergängen begegnet, ist in der Regel ein Wirtschaftswald und nicht mehr der wilde und vom Menschen fast unberührte Wald der Märchen und Sagen, der Urwald, in dem Feen und Elfen ihr Wesen treiben.

Die Selbstverständlichkeit, mit der Wald über Jahrtausende hinweg als gegeben betrachtet und genutzt wurde, ließ ihn in der Anschauung der Menschen vielfach zu einem reinen Wirtschaftsobjekt werden. Der Verlust vieler anderer Aspekte des Waldes wird uns erst seit kurzem mehr und mehr als Verarmung bewußt. Die voranschreitende Abholzung der Tropenwälder oder die schon wieder in Vergessenheit geratenen neuartigen Waldschäden in Mitteleuropa, besser bekannt unter dem Begriff "Waldsterben", sind die materiellen und sichtbaren Belege dieser Verarmung. Der zunehmende Verlust der geistigen wie auch seelischen Bezüge des Menschen zum Wald und zu den Lebensformen, die er ermöglicht, veranlaßt uns, über frühere Formen dieser Beziehung nachzudenken.

\section{Wald - ein kulturell gestaltetes Element unserer Umwelt}

Die Reduzierung der Sichtweise des Waldes auf seine wirtschaftliche Bedeutung, vor allem als Holzlieferant, hat global u. a. zu seiner Gefährdung beigetragen. Denn wer Wald sagt und dabei in erster Linie das Holz meint, gefährdet mit dieser Einstellung den Wald als Ganzes. In Anbetracht der weltweiten Verringerung der Waldbestände, der neuartigen Waldschäden und der immer vehementer geführten Diskussionen über Natur- und Umweltschutz sind wir mehr denn je zur Besinnung und Definition eines zeitgemäßen und global gültigen Waldverständnisses aufgerufen. Die Verarmung der kulturellen Bezüge der Gesellschaft zum Wald und die Erosion von Sozialstrukturen führen seit langer Zeit zu einer materiellen wie auch geistigen Beeinträchtigung des MenschUmwelt-Verhältnisses im allgemeinen und der Wälder im besonderen.

Die Geringschätzung von kulturellen Aspekten an den natürlichen Elementen unserer Umwelt und ihre gleichzeitige Aufwertung als rein wirtschaftliche Ressource schaffen eine Distanz der Menschen zu dem, was sie umgibt. So wurden auch die Wälder zu einer entzauberten Ware. Um das Wahre am Wald für uns wieder zu entdek- ken, müssen wir die Ware, die wir in ihm sehen, ein wenig in den Hintergrund treten lassen. Eine neue Anschauung des Waldes, der, weil er uns zuwächst, lange als gegeben hingenommen wurde, ist notwendig und wichtig geworden. Wir müssen den Wald anders sehen lernen und wissen, warum dies notwendig geworden ist. Die Anschauung des Waldes bedarf heute einer gesellschaftlich begründbaren Theorie seiner Anschauung.

\section{Mensch, Wald und Mythos}

Vor der Zivilisationsgeschichte des Menschen liegt seine anthropologische Entwicklungsgeschichte, die in ihrem seinsgeschichtlichen Werdegang Wald und Mensch gleichermaßen prägte. Als prominenteste unter allen anderen Waldnutzern erwerben und tradieren Menschen Wissensbestände, die ihnen in ihrer Kombination und mehr oder weniger systematischen Anwendung einen Vorteil vor anderen Lebewesen sichern.

Die Menschen der Frühzeit bewohnten in erster Linie die Waldränder und -lichtungen und nur zeitweise oder gelegentlich das Innere des Waldes. Auf den Lichtungen erschien die Beute, und im Wald selbst mußten die Sammelgüter gesucht und zusammengetragen werden. In dieser Welt von Wäldern und Lichtungen ereignet sich das, was wir die Formung der frühen Kulturen nennen. Das Vorhandensein zweier Lebensräume und die Erkennung und Zurkenntnisnahme des Unterschieds zwischen dem Wald und den überschaubaren Flächen der Flußauen, Blößen und Lichtungen als Unterschied ist ein wichtiges Moment der Kulturentfaltung. Die Optionen zweier Räume zu haben, die verschiedene Lebensformen ermöglichen und schließlich gar bedingen, bedeutet, sich darauf einzustellen, mit den spezifischen Verhältnissen dieser Räume umzugehen. Die Möglichkeit, etwas anderes als kulturellen Kosmos zu erleben als den Wald, wurde für Jäger- und Sammlergemeinschaften in diesem Prozeß erst denkbar.

Die Bewußtwerdung der Ambivalenz des Waldes als Raum des Schutzes und der Bedrohung kann als ein weiterer kultureller Entwicklungsfaktor gelten. Die Erfah-

Klaus Seeland, Dr., Soziologe, Professur für Forstpolitik und Forstökonomie, ETH-Zentrum, 8092 Zürich 
rung, daß dem Menschen kein spezieller Lebensraum eignet, sondern jeglicher ihm bekannter Raum seine bedrohlichen und schützenden Aspekte gleichermaßen bietet, verlangt nach einer unterscheidenden Ordnung. Diese Ordnung nimmt der Mensch zum Teil aus Nützlichkeitserwägungen und zum Teil als von höheren Instanzen verfügte oder begünstigte an. Die Geschichte der Raumstrukturierung durch den Menschen ist somit wesentlich eine Geschichte der ihn leitenden Ordnungsvorstellungen in Räumen, die von ihm ambivalent erlebt werden.

Raumordnung als sozio-kulturelles Vorstellungsmuster hat zwei Grundvoraussetzungen: erstens muß sie in der empirischen Erlebniswelt der Menschen wurzeln, diese gleichzeitig aber auch sinnhaft transzendieren, d. h. meist religiös deuten. Erst dann erfüllt sie die zweite Grundvoraussetzung, nämlich die, dem Legitimitätsanspruch der Gemeinschaft als dem Bedingungsfaktor für ihr Bestehen und Überdauern zu genügen. Ein bestimmter besiedelter Raum wird zum gottgegebenen oder politisch beanspruchten Ort des Wohnens, der Souveränität und territoriale Integrität behauptet. Die Wahrnehmung unterschiedlicher und dadurch auch unterschiedlich geordneter und genutzter Räume ist kulturstiftend. Der Vorzug eines Lebensraumes vor einem anderen muß für den frühen wie den heutigen Menschen begründbar und begründet sein.

Als Ausdruck dieser legitimatorischen Notwendigkeit gründet der frühe Mensch seine Ordnungsmuster zum Teil auf die naheliegende Macht der Roheit und Stärke, zum Teil auch auf höhere Instanzen. Das Pathos und die Erhabenheit der Distanz einer überirdischen Macht und die Unterwerfung unter das ganz Andere scheinen ihm die Gewähr dafür zu bieten, für Menschen verbindlich zu sein. Das Naheliegende prägt seine Lebensumstände wie z. B. die Machtverhältnisse in seiner Horde, die konkrete Ausstattung seines Lebensraums mit Dingen, die für ihn zum Lebensunterhalt notwendig sind. Beeindruckend für ihn ist aber weniger das Anwesende als vielmehr das Abwesende oder sich Verbergende und nur hier und da Zeigende oder indirekt Mitteilende. Die Gestirne, die Götter, die Toten, die Geister erlegter Tiere usw. sind in ihrer abwesenden Anwesenheit sinnmächtig und ritualstiftend. Das heißt, die dem unmittelbaren Leben fernen Dinge geben jenen Daseinsverhältnissen, aus denen heraus die Frage nach Ordnung gestellt wurde, ihren Sinn. Eine Sicherung und Verfügbarmachung der Anwesenheit des Abwesenden läßt sich aber erst durch Sprache und die verschiedenen Gattungen, in der sie gebraucht wird, herstellen (z. B. als Erzählung, Gebet, als Mitteilung, An-Rede oder Beschwörung usw.).

\section{Mythos und Wahrheit}

Der Mythos ist in erster Linie als Ursprungsmythos der Götter, der Menschen und all dessen, was ist, zu verste- hen. In der altgriechischen Kultur ist der Mythos, wie er z. B. durch Homer oder Hesiod mitgeteilt wird, die wahre Geschichte schlechthin. ${ }^{2}$ Es gibt nichts, was wahrer wäre im Sinne einer Erklärung der Ursprünge alles Seins. Mythos ist hier gleichbedeutend mit Wahrheit. Er ist eine, nach heutigen Maßstäben gemessen, vielleicht beliebige Geschichte, die mit dem Anspruch auftritt, Herkunft wie Zukunft des Seienden zu erzählen und damit auch zu deuten. Jedoch nicht immer ist der Mythos erhellend. Wie die Quelle, aus der er seinen Ursprung nahm, ist seine Aussage oft dunkel und vieldeutig. Der Mythos stellt sich als Erzählung dar, verrätselt sich aber, was seine Deutung anbelangt. Die Tatsache, daß ein Mythos vieldeutig ist, heißt in erster Linie, daß er sich grundsätzlich einer eindeutigen und eindimensionalen Aussage entzieht und vielleicht entziehen will. Deutungsversuche, die in ihm nach einem Sinn suchen, aber nur einen nicht finden können, müssen zwangsläufig scheitern. Sie finden viele Deutungsmöglichkeiten in meist vielfältigen Varianten. Beim Mythos gilt alles nur erdenklich Ableitbare aus dem Erzählten als zulässige Deutung. Es sind weder Fehldeutungen noch irrige Auslegungen denkbar. Ist der Mythos demnach wahrer als der Logos? Ist das Mehrdeutige mächtiger als das Eindeutige? ${ }^{3}$

Des Rätsels Lösung ist vielleicht im Anspruchsniveau dessen zu suchen, was erzählt wird bzw. gedeutet werden soll. Der umfassende Deutungsanspruch des Mythos muß in seinen Aussagen aus zwei Gründen unbestimmt bleiben. Zum einen ist er Ausdruck des höchsten Seinsgrundes, der durch die Erzählung zur Anwesenheit verfügt wird. All das, was war, ist und sein wird, beansprucht in dieser Erzählung kosmische Geltung. Zum anderen ist es ein allgemeiner Ausdrucksmodus sakraler Texte, sich unspezifisch mitzuteilen und so ein Verlangen nach Auslegung zu schaffen. Diese Auslegungen, aus profanen Ansprüchen heraus formuliert, bedürfen entsprechend sanktionierter Gelehrter oder Geweihter, die jene Mehrdeutigkeiten auslegen. Hier wird der Mythos zur Mythenlehre, zur Mythologie. All jene, die den Mythos in seiner sich verrätselnden Mehrdeutigkeit nicht hinnehmen wollen, sich zur Ausdeutung aufgerufen fühlen und über ihn räsonieren, verlieren dabei das Wesentliche des Erklärungsgehalts, den der ungedeutete Mythos in sich trägt, vorausgesetzt, er wird naiv aufgenommen. Wenn wir heutzutage also vom Mythos sprechen, so sprechen wir aufgrund unserer vernunftmäßigen Prägung und erkenntnismäßigen Distanz zu ihm in mythologischer Perspektive über ihn und nicht in der Annahme dessen, was von ihm als wahr mitgeteilt wird. Vom Mythos zu sprechen ist uns also nur in Form eines Beitrags zur Mythologie als einer Wissenschaft möglich. Der Mythos selbst ist für uns seit Platon eine unwahre Geschichte. So wird dieser Begriff auch heutzutage in der Alltagssprache benutzt. Der Mythos bedeutet dem frühen Menschen alles, der heutige Mensch hingegen sieht in ihm eine Falschaussage. Er interessiert sich für die Mythologie, weil er sich den Weg verstellt hat, die Wahrheiten des Mythos als für sich mögliche Wahrheiten gelten zu lassen. 


\section{Vom Wald zum Feld}

Der Wald erschließt sich von jeher in seiner Existenz und in seiner Leben gewährenden Seinsweise. In den kosmogonischen Ursprungsmythen erscheint er und in den anthropogonischen Mythen ${ }^{4}$, die von der Entstehung des Menschengeschlechts, z. B. aus einem Baum, erzählen. Im Wald als einer kosmisch vollständigen Welt versammelt sich alles, was ist. Die Erfahrung der Lichtung, des Waldrands als überschaubare Flächen macht den Wald zum diskreten Raum. Aus der oben angesprochenen erkannten Verschiedenheit von Wald und Lichtung wächst mit der Verlagerung menschlicher Aktivitäten vom Wildbeutertum zum Ackerbau die geistige Distanz zum Wald als einer Welt. Die Rodung wird zur Welterbauung, und die Welt des Waldinneren wird nunmehr zum Bereich der Unheimlichkeit. Felder bzw. Wiesen sind Räume, in die menschliche Existenz hineinwirkt, doch bleibt dem Wald sein Vorrang als ursprünglicher mythischer Raum erhalten. ${ }^{5}$ Als solcher ist er der Zwischenraum zwischen Himmel und Erde, der Versammlungsraum göttlicher und irdischer Wesen, die hier einander begegnen. ${ }^{6}$

Das Feld als bereitete Kulturfläche ist eine spätere Abwandlung eines vordem bewaldeten oder waldumringten Gebietes. Hier sind die Götter der Erde und Fruchtbarkeit anwesend, die durch ihre besonderen Zuständigkeiten für das Gedeihen der Feldfrüchte ortsgebunden und nach je spezifischen Kulten verehrt werden. So wurde zum Beispiel im lateinisch geprägten Europa im Übergang von den frühen Formen der Waldnutzung zur Akkerwirtschaft Diana, die Göttin der Jagd und des Waldes, auch zur Fruchtbarkeitsgöttin der Felder (vgl. FRAZER: 139 ff.). Informanten berichteten Tacitus z. B., daß im germanischen Siedlungsgebiet Vegetationsdämonen in einer Prozession aus einem heiligen Wald herausgefahren wurden (MANNHARDT $1: 574$ ff.) und Verwendung im Akkerkultus fanden.?

In der Entwicklung der frühen Jäger- und Sammlerkulturen zu Agrarkulturen wird deutlich, daß der Wald in seiner mythischen Ausstrahlung und physischen Ausdehnung immer mehr zurückgedrängt wird. In ihrem Verlauf wird der Wald Mitteleuropas, ehemals Kosmos, nun zum Aufenthaltsort der nicht kultfähigen Geistwesen, der Waldgeister und Dämonen. Der Kultus, welcher neue, agrarische Götter in Hainen, auf Freiflächen, in Tempeln verehrt und somit an einen Ort bannt, blickt mit Schauder auf die "freischwebenden" Waldgötter und Dämonen zurück. Die Abwendung vom Wald als einer Universalsphäre von Göttern und anderen, auf die das Leben des frühen Menschen im wesentlichen orientiert war, bedeutet eine Akzentverschiebung von der Welt der Angewiesenheit einer vor allem extraktiven Wildbeuterwirtschaft hin zu einer Form der Feldbauwirtschaft.

Der Brandrodungsfeldbau z. B. favorisiert so durch einen gewollten menschlichen Eingriff in das Waldganze das Wachstum bestimmter ausgewählter Nahrungspflanzen. Dieser Eingriff in das Ensemble des Waldes ist nur vor- übergehend, und die Produktivität dieser Rodungsinseln ist eher gering. Das Bedeutsame dieser Tatsache für den frühen Menschen ist die Erfahrung der Gleichsamkeit von Wachstum und Zerstörung. Die Wandlung eines Waldstücks in eine agrarisch zu nutzende Lichtung mit Hilfe eines Feuers bedingt die Zerstörung der vorhandenen Waldvegetation. Diese Rodungsinseln bestehen nur eine Zeitlang, bevor sie wieder in ein vegetatives Ganzes zurückfallen. Die unregelmäßig runden Rodungsinseln sind noch weit davon entfernt, zu rechteckigen Ackerflächen zu werden. Die Fähigkeit zur Unterscheidung zwischen dem allumfassenden Wald und einem vom Menschen bestimmten Raum muß als zu vollbringende und überhaupt vollbringbare, machbare Leistung erfahren werden. Die Erfahrung der Reintegration seiner Leistung durch das Ganze des Waldes zeigt dem frühen Menschen, daß Wachsen und Zerstören einander bedingende Prozesse sind.

\section{Die Macht des Menschen und die Kraft des Waldes}

Der Schritt von der Wildbeuterei zur Hege und Pflege von Tieren und Pflanzen geht einher mit der Zuschreibung von Eigentum an Land und der Identifizierung von Arbeitskraft als eines Wirtschaftsfaktors. ${ }^{8}$ War der Wald mit seinen Möglichkeiten für den frühen Menschen einer der gegebenen Horizonte seines Lebens, so wird dieser Horizont nunmehr durch die agrarische Betätigung selbst gesetzt. Die Kraft des Waldes, die sich in dem Vermögen ausdrückt, menschliche Kultivierungsleistungen wieder im Waldganzen aufzuheben, wird erst mit der permanenten Bewirtschaftung von Rodungsflächen deutlich erkennbar. Die Inwertsetzung des vom Wald befreiten Bodens ist durch die entsprechenden Arbeitsleistungen des Rodens und Erschließens mit der Behauptung von Ansprüchen permanenter, d.h. auch vererbbarer Nutzungsrechte verbunden. Der Begriff des Eigentums an Boden, der sich hier gegen einen vielleicht ursprünglicheren Begriff des Gemeinnutzens eines nicht anzueignenden, aber damit auch nicht freien (!) Gutes (des Waldganzen) durchsetzen mußte, bedurfte in erster Linie einer Legitimation und eines dauerhaften Schutzes durch Übereinkunft oder Gesetz.

Schon früh gebieten magisch-religiöse Praktiken über das Wachstum der Feldfrüchte, vor allem durch die Anwendung entsprechender Opferrituale. Priesterkönige fungieren hier als Hüter und Beförderer der Fruchtbarkeit (s. FRAZER: Kap. III-VIII, bes. VI). Von verschiedenen sozialen Gruppierungen ausgeübte Feld- und Waldkulte werden als solche überhaupt erst möglich durch die Unterscheidung von Feld und Wald und etablieren sich in einer spielerischen Vielfalt. Politische Legitimation und Machtausübung innerhalb dieser Vielfalt leiten sich von der Sicherstellung dieser gesellschaftlich bedeutsamen Feldfruchtbarkeit ab, die innerhalb einer mit und vom Wald lebenden Gesellschaft in dieser Ausprägung kaum hätte entstehen können. Der Übergang von der Nutzung 
des aus sich heraus geschehenden Waltens des Waldes zum Gestalten des Feldes bedarf einer Organisation und sozialer Strukturen. Sie nimmt im Erkennen des spezifischen Milieus und der sozialen Etablierung einer Teilung von Macht und Arbeit ihren grundsätzlichen Anfang. Der Rodung als Entstellung einer Lebenswelt, mit der der Mensch bislang lebte, kommt in diesem Prozeß die Bedeutung zu, Ursprung für den Aufgang neuer Lebensformen und -welten zu sein. Dem Wald als dem Inbegriff der Fülle eines natürlich seienden Nahrungsmittelvorrats treten die Möglichkeiten des nicht spezifisch geprägten, der leeren Fläche des Bodens entgegen. Während der Wald einem als Urwald zuwächst, muß der Anbau von Nahrungsmitteln mühevoll betrieben werden. Das Erkennen der Bedeutung leerer Flächen in ihrem Potential als zu nutzende Flächen setzt ihre Schaffung und Erhaltung bzw. Unterhaltung voraus. Diese Erkenntnis ist in ihrer Zeit eine eigentliche Kulturleistung.

Bäuerliche Lebensformen regeln mit ihrer spezifischen Arbeitsteilung in Familie und Gesellschaft und Agrargesetzen den Umgang mit kultiviertem Land und werden zur Basis sozialer Organisation. Der Wald verliert seinen kosmischen Anspruch und viele seiner numinosen Qualitäten, behauptet jedoch über die Jahrhunderte hinweg seinen Platz in kultureller wie wirtschaftlicher Hinsicht als ursprünglicher Raum. In Literatur und Dichtung, als zeitweiliger zweckgerichteter Aufenthaltsort des Menschen in Beruf und Freizeit und als Rohstofflieferant für Industrie und Gewerbe beweist er seinen Bestand und seine Geltung noch heute.

\section{Das Wesen des Waldes}

Wald ist, wie gezeigt wurde, ein vom Menschen genutztes und gedeutetes Phänomen. Seine Gestaltung als Teil der Kulturlandschaft ist ein gesellschaftlich vermittelter Prozeß. Der Wald, an den Einzelinteressen der wirtschaftlichen Nutzung, des Schutzes, der Erholung und Bildung herangetragen werden, gibt uns keine Auskunft über sein Wesen. Es ist nicht in diesen Einzelaspekten des Nutzwaldes, des Schutz- oder Erholungswaldes zu finden. Das Wesen des Waldes ist das, was in ihm und durch ihn lebt. Sein Wesen ist es, durch sein Dasein eine Fülle unspezifischer Möglichkeiten zu gewähren. Als charakteristischer Raum gewährt Wald alle in ihm möglichen Lebensformen. Er bietet vielen Lebewesen die notwendige Fülle für die Lebensgestaltung ihres Daseins. Das Walten dieser Fülle im ständigen Wandel ist das Wesen des Waldes. Und das meint nicht nur den Wandel der Vegetation zu den Jahreszeiten. Gemeint ist hier auch die gesellschaftliche Sichtweise des Waldes im Wandel der Interessen, die an ihn herangetragen werden. Das Wesen des Waldes und die besondere Art seines Seins besteht im Wachsen und Walten. Das Wirken des Menschen greift in diesen Prozeß gestaltend ein und schafft damit ein kulturbedingtes Wirkungsganzes. Das Kulturbedingte, das im Laufe der Zeit Gewordene und Umgeschaffene am Wald, was wir z. B. als Historiker an der heutigen Form der Wälder ablesen können, ist die Art und Weise eines spezifischen Umgangs einer Gesellschaft mit dem Wald in der Geschichte. Jegliche Eingriffe darin waren immer räumlich und zeitlich begrenzt. Wald war und ist immer nur das, was der Mensch als Wald zugelassen hat.

Die Bedeutung des Waldes ist Ausdruck eines kulturell geprägten Verständnisses. Sie wandelt sich mit der Zeit, und jede der vielen Kulturen unserer Erde mißt ihm eine andere zu. Wald ohne jegliche Bedeutung gibt es heute nirgendwo. Unter der Oberfläche des Waldes, der als Wirtschaftsfaktor gesehen wird, leben noch mehr oder minder kräftig die Wurzeln von Auffassungen, die ihm andere Bedeutungen zuweisen. Auch entstehen aus diesen Wurzeln wieder Bedeutungen, die schon als abgestorben und verloren galten. Und dies gibt uns den Hinweis, daß der Wald durch die naturethischen Prinzipien des Wachsens und Überdauerns bis zum heutigen Tag seine Geltung als gesellschaftlich und kulturell bedingte Formation bewahrt hat.

\section{Waldbewirtschaftung als Kulturaufgabe}

Die Bewirtschaftung des Waldes ist ein Wirken, an dem der Mensch teilhat. Der frühe Mensch hat den Wald als Ganzes belebt; Wald war ein wichtiger Teil seiner Welt. Und auch heute wird er ihm nur durch die Zurkenntnisnahme dieses Wesens des Waldganzen gerecht. Die moderne Waldbewirtschaftung zeichnet sich durch eine Entwicklung der historisch überkommenen Sichtweise und eine weiter fortgeführte Umprägung des Waldbestandes aus. Eine Würdigung der Leistungen des Gesamtwaldes muß nach heutiger Rationalität eine Bewertung des Waldganzen vornehmen und Kriterien dafür finden. Die Vermarktung einzelner Aspekte des Waldes in Form von marktfähigen Gütern und Leistungen wie z. B. der Holznutzung, des Schutzes vor den Auswirkungen von Naturereignissen sowie der Erholung und Bildung stehen hier im Blickpunkt des wirtschaftlichen Interesses. In der Zuschreibung von Funktionen an den Wald findet eine Umprägung des natürlichen Waldganzen nach wirtschaftlicher Maßgabe statt, und zwar dadurch, daß einzelne Leistungen und Funktionen gegen andere wirtschaftlich bedeutsame bzw. immaterielle Werte aufgerechnet werden oder zumindest aufgerechnet werden können. Wenn die Leistungen des Waldes in bezug auf seinen Ertrag aus der Holznutzung gegen die Berechnung seiner Erholungsleistungen, die momentan vielleicht höher veranschlagt werden, aufgewogen werden, dann geht Erholungswald vor Nutzwald. Werden dem Erholungswald irgendwann jedoch Schutzwirkungen zugeschrieben, die ihrerseits den errechneten Wert des Erholungswaldes übersteigen, erhält der Schutzwald Vorrang vor dem Erholungswald. Dieses Beispiel ist fiktiv, aber nicht undenkbar. Es macht, wie ich meine, das Dilemma des wirtschaftlich bzw. monetär orientierten Interesses an materiellen wie immateriellen Leistungen des Waldes deutlich. 
Die Eigenart des Waldes jedoch bleibt aber nur erhalten, wenn sie als positiver Wert politisch gesetzt wird. Dem Bestand des Waldes als kulturgeschichtliches Phänomen wird nur somit Geltung verschafft. Erst die generelle politische Anerkennung des Waldganzen kann einzelnen wirtschaftlichen und ästhetischen Nutzungsaspekten die ihnen zukommende relative Bedeutung zuweisen. Errechnete Wertrelationen verschiedener Waldleistungen pro Kopf der Bevölkerung dürfen im politischen Kräftespiel nicht gegeneinander ausgespielt werden. In erster Linie ist Wald Wald und hat gleichzeitig alle Funktionen. Wertberechnungen können durchaus angestellt, müssen aber als sekundäre Aspekte gesehen werden. Das hier in Erscheinung tretende Grundproblem ist, daß dabei politische und wirtschaftliche Rationalität nicht in einen Gegensatz geraten dürfen, d.h., daß politische Dogmen nicht vermeintliche Unwirtschaftlichkeit decken dürfen. Was aber Unwirtschaftlichkeit in diesem Kontext heißt, muß auch wiederum neu überdacht werden.

Die Erkenntnis und gesellschaftspolitische Anerkennung des unschätzbaren Gesamtwertes des Waldganzen steht vor jedem berechenbaren Partikularinteresse. Solange die Waldbewirtschaftung nur ein Teilaspekt ist und die grundsätzliche Erhabenheit des Waldganzen anerkennt, werden damit die Ansprüche an den Wald sowie auch die Funktionszuschreibungen an ihn relativiert. Wenn das Waldganze nicht nur aus forstpolitischer Perspektive, sondern aus allgemein politischer Optik im Denken und als Übereinkunft in der Gesellschaft ungeteilt bleibt, ist es als Ganzes gewonnen.

\section{Wald - ein Aspekt der Gesellschaft}

Wesentliche Teile unserer Wälder sind und werden in absehbarer Zeit forstlich bewirtschaftete Flächen bleiben. Die Erhaltung und Nutzung des Waldes aus der Sicht des Waldganzen als kulturelle Aufgabe wird aber zunehmend an das Bewußtsein auch forstlicher Kreise drängen und dort vermutlich entsprechende Wirkungen zeitigen. Die Bewirtschaftung von Teilaspekten des Waldes und seine Bewertung nach Funktionen werden dann vielleicht die Enge ihrer reinen Verwertungsperspektive überwinden können. So kann es möglich werden, zu neuen physisch-ökologischen wie soziokulturellen Rahmenbedingungen zu gelangen, die in Wechselwirkung mit politischen Prozessen stehen. Die Gesamtsicht des Waldganzen wird sich dann als gesellschaftspolitischer Standpunkt nicht mehr ignorieren lassen.

Vergessen wir nicht: das Strukturprinzip des Waldes ist ein sich selbst hervorbringendes (gr. poiesis). Wald bringt sich selbst hervor, er gestaltet sich in diesem Sinne grundsätzlich "poietisch», d. h. im virtuosen Umgang mit dem Zustand, in dem man ihn gewähren läßt. So bringt er sich in eine unbestimmte Form und Fülle, die der Mensch dann für seine Zwecke umschaffen kann. Die Waldbewirtschaftung ist ein Modus des Menschen in der modernen Gesellschaft, sich ökonomisch zum Wald zu verhal- ten. Sie ist infolge der forstlichen Waldfunktionenlehre und durch staatliche Subventionspolitik eine Wirtschaftsform. Mittelfristig ist sie in die Ressourcen- und Raumplanung zu integrieren und wird auch in Zukunft ein prägendes Kulturphänomen sein. Eine Kulturaufgabe für die Zukunft besteht in dieser Hinsicht nun unter anderem darin, im spezifischen Sein des Waldganzen und seiner Erhaltung einen gesellschaftlichen Wert zu erkennen, der richtungsweisend für die Übernahme breiterer Aufgaben sein kann, ohne auf Waldbewirtschaftung in großem Umfang zu verzichten.

Eine moderne Gesellschaft verhält sich zu ihren spezifischen Räumen wie Stadt, Wald, Landschaft, Fluß, See und Berg wie zu den sozialen Gruppierungen, aus denen sie besteht. Alle sind in ihrer gesellschaftlichen Bedeutung in der Planung und Gestaltung berücksichtigt, die die Charakteristik eines Landes ausmachen. Unter Wahrung dieser Eigenschaften und Identitäten werden ihnen Möglichkeiten zur Selbstgestaltung zugebilligt. Waldbewirtschaftung ist in diesem komplexen Zusammenhang die der Gesellschaft zugekehrte, heute meist nur wirtschaftliche Seite der Gestaltung eines Landes. Die ganze Breite des Horizontes einer Waldnutzung in ihrer gesellschaftlichen Bedingtheit und zunehmenden Vielfalt zu sehen heißt, um Sichtweisen seiner uns noch abgewandten Seiten bemüht zu sein. Diese Weite des Horizontes wird u. a. durch die Bemühungen der Waldpädagogik gekennzeichnet, die zwischen Wald und Mensch vermitteln will.

\section{Durch den Wald zur Natur}

Der Weg zur Natur führt heute vielfach durch den Wald. Die Waldnähe, die seit geraumer Zeit in der industrielltechnischen Gesellschaft verstärkt gesucht wird, findet ihren Ausdruck u.a. in der Existenz zahlreicher Naturschutz-, Umweltschutz- und Umwelterziehungsgruppen, die sich des Waldes angenommen haben. Die Vielzahl der Ansätze und die Vielfalt der Motivationen, sich mit dem Wald auseinanderzusetzen, kann als Orientierung zur Natur in pragmatischer Hinsicht gesehen werden. Doch man kann sie auch als eine neue Empfindsamkeit gegenüber der Versehrtheit der Natur verstehen. Der Wald wird oft als Stellvertreter der Natur empfunden, der sich als stadtnahe Gegenwelt darstellt. Er gilt vielen als Ergänzungsraum für die technisch bestimmte Alltagswelt und als Lehrpfad der Sinne, der Naturqualitäten vermitteln soll. Die Trennung des Menschen vom unmittelbaren Erleben der Natur, die vor allem durch den heutigen Vorrang der Medien vor dem direkten Naturkontakt vorangeschritten ist, soll zumindest teilweise in der Erfahrung des Waldes als Naturerfahrung überbrückt werden. Natur ist zeitlos, doch der Mensch lebt jeweils in seiner Generation, in seiner Zeit. Auch hier nimmt nun der Wald wiederum eine Mittelstellung ein. Er ist weder ganz Natur, also ganz zeitlos, noch ist er in seiner Substanz ein gesellschaftliches Produkt. Wald ist also Natur in der Formgebung einer bestimmten Zeit. 
Die Stadtnähe des Waldes begünstigt den Zugang zu ihm, und dies ist der Formulierung einer Haltung zu ihm förderlich. Das Finden einer Einstellung zum Wald signalisiert möglicherweise die Entdeckung eines Verhältnisses zur Natur, welches dem Bürger vielleicht noch nicht bewußt ist. Dabei ist es sekundär, daß Wald ja nur ein kleiner und, seiner Seinsart gemäß, besonderer Ausschnitt aus der Natur ist. Natur drückt sich als Wirkungsprinzip aus, das im Wald und durch ihn wirkt und gestaltet - je nachdem, wie der Mensch ihre Gestaltungen zuläßt.

Der Umgang der Gesellschaft mit dem Wald stellt ihr kulturelle und politische Aufgaben, die sich im Laufe der Zeit wandeln und dadurch immer neue Lösungen von ihr fordern. Um der Interessenvielfalt in ihr zu entsprechen, muß eine pluralistische Gesellschaft ihren Wald vor allem als Ganzes sehen. Falls sie eine solche Sichtweise verlernt hat, muß sie sich diese wieder aneignen, denn viele Menschen haben viele verschiedene Ansprüche an den Wald.

Was können wir vom Wald und dem möglichen Erleben eines Teils der Natur in ihm erwarten? Kann aus einer Haltung zum Wald und zur Natur ein ethos (gr.: ein Ort des Wohnens) des Lebens werden? Ist hier ein Wissen um dieses Ethos und ein ihm gemäßes Verhalten, eine Ethik möglich? Nimmt die Nähe des Waldes und die Möglichkeit, ihn zu erleben, Einfluß auf unser ethisches Empfinden? Fühlen wir uns vielleicht deshalb von den neuartigen Waldschäden betroffen, weil wir als Teil der Natur ethisch, sozusagen an unserem Wohnort, Schaden nehmen? Dies sind bedenkenswerte Fragen unserer Zeit, auf die wir noch keine Antworten haben. Wenn wir in der Lage sind, diese Fragen zu beantworten oder sie durch treffendere zu ersetzen, um unser Verhältnis zu Natur und Wald zu klären, ist ein Beitrag zur Erhellung der uns bislang noch abgewandten Seiten unseres gesellschaftlichen Bezugs zum Wald geleistet.

\footnotetext{
Anmerkungen

' Überarbeitete Fassung eines Vortrags an der Evangelischen Akademie Baden am 8.5.1992.

${ }^{2}$ Siehe dazu GRASSI, Ernesto, Die Theorie des Schönen in der Antike. Köln: Dumont ${ }^{2} 1980$. p.163 ff. und DERS., Kunst und Mythos. Reinbek: Rowohlt, 1956.
}

${ }^{3}$ OTTO, Walter F., Gesetz, Urbild und Mythos. In: DERS., Die Gestalt und das Sein. Gesammelte Abhandlungen über den Mythos und seine Bedeutung für die Menschheit. Darmstadt 21959, p. 27-90.

${ }^{4} \mathrm{Vgl}$. hierzu die von MANNHARDT, Wilhelm, Wald- und Feldkulte. 2 Bde. Berlin: Gebr. Borntraeger ${ }^{2} 1904 / 05$, Bd.1, p. 6 ff., angeführten Beispiele des Bundehesch-Mythos der AltIraner, der nordamerikanischen Indianer (namentlich Sioux, Karaiben und Antillenindianer), des germanischen Askr und Embla-Mythos sowie Beispiele aus den Mythen und Sagen, die DÄHNHARDT gesammelt hat: DÄHNHARDT, Oskar $(\mathrm{Hg}$. Natursagen. Eine Sammlung naturdeutender Sagen, Märchen, Fabeln und Legenden. 4 Bde. Leipzig/Berlin: Teubner, 1907, Bd. I, pp. 4, 8, 28 f., 34 f., 126, 178, 183.

${ }^{5}$ Nach den Ausführungen Giovanni Battista VICOs z. B., der in seinen Prinzipien Einer Neuen Wissenschaft - Über Die Gemeinsame Natur der Völker ('1725 u. ö.) z. B. die Beschreibung Germaniens von Tacitus anführt, sagt, "daß sie [die Germanen, K. S.] ihre Götter an heiligen Stätten anbeteten, die er 'lucos et nemora' 'Lichtungen und Haine, nennt; das müssen gerodete Stellen innerhalb der geschlossenen Fläche der Wälder gewesen sein ..." (Zif. 479) haben die Germanen ihren Göttern nicht an deren Aufenthaltsorten im Wald gehuldigt, sondern auf freien "kultivierten" Freiflächen. Des weiteren führt Pausanias in seiner Beschreibung Griechenlands an: "... denn man lichtete die Wälder, um den Ausblick zu haben für die Betrachtung, von wo die Auspizien der Adler herkamen, die höher fliegen als alle Vögel." (Zif. 480). VICO berichtet die Schilde der barbarischen Zeiten, die rund waren, "weil die entwaldeten und bebauten Ländereien die ersten 'orbes terrarum, "Erdkreise, waren..." (Zif. 564), und in der römischen Mythologie heißt es: "...Vulkan [der Feuergott, K. S.] hatte Feuer an die Wälder gelegt, um bei offenem Himmel zu beobachten, woher Jupiter seine Blitze senden würde." (ebenda).

${ }^{6}$ Vgl. FRAZER, James George, The Golden Bough. A Study in Magic and Religion. Abriged Ed. in 1 vol. New York: MacMilIan, 1944 Kap. I (The King Of The Wood), IX (The Worship Of Trees), XV (The Worship Of The Oak).

${ }^{7} \mathrm{Vgl}$. dazu den römischen Mithra-Kult, in dem durch das jährliche Stier-Blutopfer auf dem Ackerfeld die Fruchtbarkeit, die dieses Tier allgemein symbolisierte, mit Hilfe seines Blutes auf die Wachstumskraft des Korns übertragen wurde (s. CUMONT, F., Die Mysterien des Mithra. ${ }^{3}$ Leipzig/Berlin: 1923 zit. in: SCHWABE, J., Archetyp und Tierkreis, p. 37).

${ }^{8}$ Anregende Ausführungen hierzu gibt IMMLER, H. Vom Wert der Natur. Zur Ökologischen Reform von Wirtschaft und Gesellschaft. Opladen: Westdeutscher Verlag, 1989, pp. 96-147, bes. pp.119 ff. 